\author{
Felicja Księżyk \\ ORCID: 0000-0002-6913-0108 \\ Uniwersytet Opolski, Opole
}

DOI: $10.19195 / 0435-5865.143 .16$

\title{
Warum Faszinierendes im Polnischen 'intrigiert'? Von verbalen falschen Freunden im Sprachenpaar Deutsch-Polnisch
}

\begin{abstract}
Die sog. falschen Freunde werden bekanntlich als potentielle Interferenzquelle bei Übersetzern und im Fremdsprachenunterricht betrachtet. In der einschlägigen Literatur zum Sprachenpaar Deutsch-Polnisch werden sie mit Vorliebe durch substantivische Wortpaare veranschaulicht. Der vorliegende Beitrag widmet sich verbalen falschen und wahren Freunden, wobei insbesondere bei wahren Freunden nicht nur Bedeutungsgleichheit, sondern auch eventuelle Verwendungs- bzw. Usualitätsunterschiede zu berücksichtigen sind.
\end{abstract}

Schlüsselwörter: falsche und wahre Freunde, verbale falsche Freunde, Usualität

\section{Why, what is fascinating, in the Polish language intrigues? About verbal false friends of a translator in the Polish-German combination}

The so-called false friends are known to be a potential source of interference in translation and foreign language learning. In the relevant literature concerning linguistic Polish-German pairs, false friends are often illustrated on the example of noun pairs. This article is devoted to false and true verbal friends when, especially in the case of true friends, not only convergence of meaning is taken into consideration but also possible differences in their use or alternatively their usualness.

Keywords: false and true friends, verbal false friends, usualness

Felicja Księżyk, Uniwersytet Opolski, Instytut Filologii Germańskiej, Pl. Staszica 1, 45-052 Opole, Polen, E-Mail: ksiezykf@uni.opole.pl

Received: 22.09.2017, accepted: 18.05.2018

Germanica Wratislaviensia 143, 2018

(C) for this edition by CNS 


\section{Einleitende Bemerkungen}

Bei der Frage, ob die sog. falschen Freunde des Übersetzers ausreichend untersucht worden sind, scheint in der Forschung kein Einklang zu bestehen. Während diese Frage von Chodacka und Schöffel eindeutig bejaht wird:

[den] ,Faux amis' [wurde] unseres Erachtens zumindest für den Fremdsprachenunterricht zu große Aufmerksamkeit geschenkt [...]. Dieses Gebiet der Tautonyme ist bereits gründlich untersucht und die Ergebnisse [sind] publiziert worden [...] (Chodacka/Schöffel 2000: 171)

vertritt Kuczyński eine geradezu gegensätzliche Position:

Obwohl die sprachwissenschaftliche Erforschung der falschen Freunde noch unbeleuchtet bleibt, sind bisher viele Berufsübersetzer in die von ,faux amis' gestellte Falle gegangen. (Kuczyński 2003: 255)

Auch wenn zu dem Phänomen der falschen Freunde bereits viel publiziert wurde, erscheint es notwendig - da Sprachen im ständigen Wandel begriffen sind die Bedeutungsrelationen zwischen den analysierten Wortpaaren, wie sie in den einschlägigen Wörterbüchern verzeichnet sind, zu revidieren. Während etwa 'konkurs' im Altpolnischen durchaus auch gemäß der Bedeutung des deutschen Nomens 'Konkurs' d.h. im Sinne von 'Insolvenz' gebräuchlich war (vgl. Księżyk 2015: 335), und auch in den einschlägigen Tautonymenwörterbüchern diese Bedeutungsvariante mit der Markierung selten, bzw. veraltet verzeichnet wird, ergibt die Recherche im Nationalkorpus der Polnischen Sprache (NKJP/www.nkjp.pl), dass diese Bedeutung im Gegenwartspolnischen nicht mehr aktualisiert wird: im NKJP erscheint es stets nur im Sinne eines Wettbewerbs bzw. einer Ausschreibung. Somit dürfte das Wortpaar dt. Konkurs - pl. konkurs nicht mehr als Beispiel für partielle falsche Freunde, sondern vielmehr als eines für absolute falsche Freunde betrachtet werden.

Der Begriff „falsche Freunde des Übersetzers“ ist bekanntlich eine Lehnübersetzung des französischen Ausdrucks ,faux amis du traducteur“, der erstmals 1928 von Maxime Koessler und Jule Deroquigny verwendet wurde (vgl. Kuczyński 2005: 43 und Lisiecka-Czop 2007/2008: 272). Nach Lipczuk sind in der einschlägigen Literatur folgende konkurrierende Termini zur Bezeichnung des Phänomens der falschen Freunde im Umlauf: „'Falsche Freunde des Übersetzers', Faux Amis, zwischensprachliche Homonyme, Inter-Homonyme, interlinguale Homonyme, interlinguale Paronyme, lexikalische Scheinidentitäten, scheinbare Äquivalente, Pseudoäquivalente, Pseudointernationalismen, irreführende Fremdwörter, Tautonyme" (URL 1). Janikowski und Krzywda (2011: 116-117) führen eine noch längere Liste von Bezeichnungen in diesem Bereich an, u.a.: Approximate, internationale Homonyme, Paronyme, Analogonyme, Pseudoanalogonyme, Entlehnungen, Kognate, Falsiäquivalente, Tautonyme, Pseudointernationalismen, Interferenten, trügerische Äquivalente, trügerische Kognate oder trügerische Wörter, Scheinäquivalente, unzuverlässige 
Freunde, primäre Äquivalentassoziationen, primary mappings, falsche Freunde, partielle falsche Freunde und sogar halbehrliche Freunde, wobei diese Bezeichnungen z.T. etwas andere Erscheinungen bezeichnen und falsche Freunde als der gängigste Terminus gelten kann. Neben diesen Bezeichnungen führt Kuczyński (2003: 259-262) zusätzlich noch Fallstricke des Wortschatzes, interlinguale Analogismen und die Relation der irreführenden zwischensprachlichen Ähnlichkeiten an. Obwohl gegen den Begriff „falsche Freunde des Übersetzers“ Einwände im Hinblick darauf erhoben wurden, dass er für einen wissenschaftlichen Terminus nicht geeignet sei, zumal das Phänomen nicht nur die Übersetzertätigkeit betrifft, hat er sich dennoch durchgesetzt (vgl. Lipczuk 1992: 139); als einen geeigneten Fachbegriff könnte man in diesem Zusammenhang den von Lipczuk geprägten Vorschlag der Tautonyme betrachten (vgl. Kuczyński 2003: 259, 262). Wie Lipczuk et al. präzisieren, handelt es sich allerdings bei Tautonymen um ein Hyponym der falschen Freunde. Gemeint sind damit Scheinäquivalente, bei denen die phonologisch-graphische Form zweier Begriffe in zwei Sprachen ähnlich bzw. identisch ist, die Bedeutung jedoch differiert (vgl. Lipczuk et al. (1995: 7). In dem vorliegenden Beitrag wird daher auf das analysierte Phänomen lediglich mit den Begriffen Tautonyme und falsche Freunde (des Übersetzers) referiert: die analysierten Wortpaare stellen Tautonyme dar und sind folglich zugleich falsche Freunde.

In der einschlägigen Literatur finden sich unterschiedliche Definitionen und Typologien der falschen Freunde (vgl. Kuczyński 2003: 262-276, Kosturek 2013: 95-96 und Grosse 1998: 361-362). Im vorliegenden Beitrag werden die analysierten Wortpaare in Anlehnung an Lietz und Bawej lediglich nach folgenden Typen unterschieden (vgl. Lietz 2006: 327-330 und Bawej 2014: 221-235):

1) absolute falsche Freunde (Kontrast) - gemeinsam ist lediglich das formale Erscheinungsbild (das Laut- oder Schriftbild), die Bedeutungen fallen jedoch in keinem Bereich zusammen;

2) partielle falsche Freunde mit folgenden Untergruppen:

a) Inklusion - ,ein Lexem weist mehr Bedeutungen als das andere auf“ (Kuczyński 2003: 265), wobei zumindest eine Bedeutungsvariante gemeinsam ist. Hierbei wird zusätzlich unterschieden, ob das Verhältnis der Divergenz oder Konvergenz vorliegt.

b) Überlappung - ,Konvergenz- und Divergenzbeziehungen werden kombiniert. Obwohl ein Kern gemeinsamer Bedeutung vorliegt, hat jede Sprache jedoch weitere Bedeutungen entwickelt" (Kuczyński 2003: 266).

Aus dem Interessenbereich fallen somit sog. formale falsche Freunde, d.h. fiktive falsche Freunde bzw. Neuschöpfungen, die aufgrund formaler Analogieschlüsse gebildet werden, da solche in dem gewählten Analysekorpus nicht vorkommen. Im vorliegenden Beitrag bedarf zudem die Begriffsbestimmung der wahren Freunde einer Präzisierung. Der in Analogie zu falschen Freunden gebildete Ausdruck der wahren bzw. echten Freunde wird für gewöhnlich als 
Bezeichnung für Wortpaare gebraucht, die formal und semantisch identisch bzw. in einem solchen Maße ähnlich sind, dass deren Verwendung in Analogie zu der muttersprachlichen Entsprechung mit keinerlei Risiko eines fehlerhaften Gebrauchs behaftet ist (vgl. Lisiecka-Czop 2007/2008: 275). Im vorliegenden Beitrag werden damit allerdings in Anlehnung an Chodacka und Schöffel auch „solche Wörter [verstanden], deren aktuelle Bedeutung im jeweiligen Kontext übereinstimmt, auch wenn in anderen Bedeutungsvarianten diese Übereinstimmung nicht gegeben ist" (Chodacka/Schöffel 2000: 171) - d.h. einige Bedeutungsvarianten der partiellen falschen Freunde, die dann insbesondere im Hinblick auf eventuelle Verwendungs- bzw. Usualitätsunterschiede durchleuchtet werden.

\section{Zum Analysekorpus}

Auch wenn das größte Ausmaß von falschen Freunden bei eng miteinander verwandten Sprachen angenommen wird (vgl. Grosse 1998: 361), weist auch das Sprachenpaar Deutsch-Polnisch vor dem Hintergrund der vielfältigen nachbarschaftlichen Beziehungen eine Menge an Tautonymen auf.

Das deutsch-polnische Tautonymenwörterbuch von Lipczuk et al. (1995) enthält 924 deutsch-polnische Wortpaare, wovon den größten Anteil substantivische Tautonyme mit 647 Belegen bilden, gefolgt von 220 verbalen Tautonymen. Das Wörterbuch verzeichnet darüber hinaus 57 adjektivische Tautonyme. Es enthält demnach im Vergleich zum ersten kleinen deutsch-polnischen Tautonymenwörterbuch von Lipczuk (1990) mehr als vierfach so viele Wortpaare aus dem Sprachenpaar Deutsch-Polnisch: Das kleine Wörterbuch führt insgesamt 195 Wortpaare auf, auch darin bilden substantivische Wortpaare mit 133 Belegen den Großteil, gefolgt von 51 verbalen und 11 adjektivischen Tautonymen.

Im vorliegenden Beitrag sollen die 220 verbalen Tautonyme aus dem Wörterbuch von Lipczuk et al. (1995) näher untersucht werden. Eine Untersuchung der verbalen falschen Freunde lieferte auch 2005 Kuczyński, er beschränkte sich allerdings nur auf 50 Wortpaare, seine Untersuchung betraf die Angabe der möglichen Bedeutungen, die zeitliche Zuordnung des jeweiligen Lexempaares, diatopische Unterschiede, Konnotationen und die Gebrauchsfrequenz (vgl. Kuczyński 2005: 81). 


\section{Untersuchungsergebnisse}

Innerhalb der 220 verbalen Tautonyme bilden absolute falsche Freunde, bei denen die Bedeutungen der Lexeme keine Gemeinsamkeiten aufweisen, lediglich ca. $13 \%$ der Belege (28 Belege), etwa:

1)

dt. variieren (Lipczuk et al. 1995: 320)

1. zmieniać się, różnicować się, wahać się:

Das Gefieder dieses Vogels variiert von gelbbis graubraun.

2. zmieniać, odmieniać, urozmaicać: Er verstand es seine Anreden zu variieren.

3. (muz.) tworzyć wariacje melodyczne, rytmiczne lub harmoniczne danego tematu: ein Lied, ein Thema variieren

\section{pl. wariować}

1. wahnsinnig werden, geistig erkranken, verrückt werden, den Verstand verlieren, um den Verstand kommen; (pot.) überschnappen: Ze strachu ludzie wariowali.

2. (pot.) seine Gefühle unbeherrscht zeigen, sich stark aufregen; wariować z niepokoju, ze zmartwienia, z radości; (frazeol.) wariować za kimś, dla kogoś (,närrisch verliebt sein, an jdm. einen Narren gefressen haben")

3. (pot.) sich wie wahnsinnig benehmen (besonders beim Spielen), tolle Streiche machen; toben: Dzieci wariowaty na podwórku.

\section{2)}

dt. zigeunern (Lipczuk et al. 1995: 328) (pot.) prowadzić cygańskie życie, wędrować, włóczyć się: Er hat durch die halbe Welt zigeunert.

\section{pl. cyganić}

(pot., reg.) betrügen, lügen, gaunern, schwindeln: Nie wierz mu, na pewno cygani.

Partielle falsche Freunde, die didaktisch größere Schwierigkeiten darstellen (vgl. Lietz 2006: 328), bilden somit mit 183 Belegen (83\%) den absoluten Großteil der analysierten Wortpaare. Neun der extrahierten Wortpaare lassen sich von der angegebenen Semantik her als wahre Freunde einstufen, wobei z.T. diatopische Unterschiede bzw. semantische Kombinationsbeschränkungen von den Autoren des Wörterbuches angegeben werden.

Innerhalb der partiellen falschen Freunde dominiert die Relation der Inklusion mit 135 Belegen (61\%). Dabei stellt sich heraus, dass hierbei die deutschen Lexeme in den meisten Fällen mehr Bedeutungen aufweisen als deren polnische Scheinäquivalente (105 Belege, d.h. 78\% aller Inklusionsfälle), etwa: 
3)

\begin{tabular}{|c|c|}
\hline $\begin{array}{l}\text { dt. konzipieren (Lipczuk et al. 1995: 154) } \\
\text { 1. planować, obmyślać, układać, zrobić } \\
\text { szkic (czegoś), sporządzić konspekt (czegoś), } \\
\text { koncypować: einen Plan, eine Idee, einen } \\
\text { Gedanken, einen Text, eine Rede, einen } \\
\text { Aufsatz, einen Rennwagen konzipieren; Der } \\
\text { Bau ist für } 10 \text { Wohnungen konzipiert. (szersze } \\
\text { znaczenie i większa częstotliwość użycia niż } \\
\text { koncypować) } \\
\text { 2. zajść w ciążę, począć }\end{array}$ & $\begin{array}{l}\text { pl. koncypować } \\
\text { (rzad., czasem: żart.) konzipieren (1), entwer- } \\
\text { fen, planen, projektieren: Wszystkie podania } \\
\text { do władz, odwołania ja mu koncypowatem, bo } \\
\text { sam nic wymyślić nie potrafit. }\end{array}$ \\
\hline
\end{tabular}

4)

\begin{tabular}{l|l}
\hline dt. denunzieren (Lipczuk et al. 1995: 155) & pl. denuncjować \\
1. denuncjować, donosić (policji itp.): Er hat & denunzieren, anzeigen, verraten ( $\uparrow$ denunzie- \\
ihn bei der Polizei denunziert. & ren 1) \\
$\begin{array}{l}\text { 2. deprecjonować, napiętnować, (publicznie) } \\
\text { krytykować, obniżać wartość (czegoś): ein } \\
\text { Buch, eine Meinung als etw. denunzieren }\end{array}$ & \\
\hline
\end{tabular}

Das umgekehrte Verhältnis der Divergenz tritt im Vergleich dazu viel seltener auf:

5)

dt. diskontieren (Lipczuk et al. 1995: 59-60) (finans.) dyskontować; realizować weksel (przed terminem płatności z potrąceniem procentu): Die Bank diskontierte die Wechsel der Baufirma.

pl. dyskontować

1. (finans.) diskontieren (Wechsel vor ihrer Fälligkeit unter Abzug der Zinsen ankaufen) ( $\uparrow$ diskontieren): Bank dyskontuje weksle.

2. ausnutzen, Nutzen (aus etwas) ziehen, eine bestimmte Situation ausnutzen: dyskontować swoje naukowe osiagnięcia, naukowy autorytet

6)

dt. intrigieren (Lipczuk et al. 1995: 116) intrygować, knuć, robić intrygi, działać podstępnie: beim Vorgesetzten gegen die Kollegen intrigieren pl. intrygować

1. intrigieren; Intrigen (gegen jdn.) inszenieren, anzetteln; Ränke schmieden, spinnen; hinterhältige Pläne schmieden ( $\uparrow$ intrigieren): Ktoś tu skrycie między nami intryguje. 2. interessieren, Interesse wecken, neugierig machen, zu denken geben: Ten czlowiek $i$ ta sprawa intryguje mnie swa tajemniczością.

Wortpaare, die als Überlappung einzustufen sind, bei denen ein gemeinsamer Bedeutungskern besteht, aber jede Sprache zusätzlich andere Bedeutungen aktualisiert, bilden in dem analysierten Korpus 48 Belege (22\%). Zur Veranschaulichung seien etwa folgende Fälle angeführt: 
dt. sozialisieren (Lipczuk et al. 1995: 285)

1. (ekon.) socjalizować, zmieniać własność środków produkcji z prywatnej na społeczną: Industrien, Wirtschaftszweige sozialisieren 2. (socjol., psych.) resocjalizować (kogoś) (działać w celu przystosowania kogoś do życia w społeczeństwie): die sozialisierende Funktion der Kunst pl. socjalizować

1. (pot.) dem Sozialismus neigen, dem Sozialismus gegenüber Sympathie bezeigen: socjalizujaca młodzież

2. (ekon.) sozialisieren, verstaatlichen, vergesellschaftlichen, in staatlichen Besitz überführen ( $\uparrow$ sozialisieren 1$)$
8)

dt. promovieren (Lipczuk et al. 1995: 241)

1. (nie: w Austrii) uzyskać stopień doktora, zostać doktorem, uzyskać promocję, doktoryzować się: Er hat an der Universität Hamburg zum Dr. med. promoviert.

2. nadać, przyznać (komuś) stopień doktora; doktoryzować, promować (kogoś): Er wurde an der Universität Hamburg zum Dr. med. promoviert, (węższe znaczenie niż promować 2)

3. (rzad.) promować, popierać, lansować, wynagradzać: ...daß Scheinheiligkeit geachtet und Schwachsinn promoviert wird. (GDW)

\section{pl. promować}

1. (einen Schüler in die nächste Klasse) versetzen: uczeń świeżo promowany do trzeciej klasy,

2. promovieren (2), (jdm.) die Doktorwürde oder einen Offiziersrang verleihen: promować kadeta na oficera

3. fördern, unterstützen, promovieren ( $\uparrow$ promovieren 3): Niewatpliwie jest to też wina prasy i telewizji, które nie chca czy nie umieja promować dobrych, ciekawych książek. (Now)

Konsultiert man die einschlägigen Wörterbücher des Deutschen bzw. Polnischen, um die Bedeutung der analysierten Wortpaare in den jeweiligen Gegenwartssprachen zu verifizieren, dann offenbart sich, dass diese Angaben teilweise aktualisiert werden müssten.

Während etwa in dem Wörterbuch von Lipczuk das Wortpaar applizieren aplikować als ein Beispiel für Überlappung gelten kann, wie die Gegenüberstellung veranschaulicht:

9)

dt. applizieren (Lipczuk et al. 1995: 26)

1. zastosować, realizować, wprowadzać w życie: Diese Denkmodelle lassen sich nicht auf unsere Verhältnisse applizieren; eine Terminologie applizieren

2. (med.) aplikować, dawać (komuś) środek leczniczy: Der Arzt applizierte ihm eine Spritze in den Unterarm. (węższe znaczenie niż aplikować 1)

3. aplikować; naszywać, nakładać aplikację pl. aplikować

1. (jdm. etw.) verabreichen, geben, (etw.) anwenden, applizieren (2): aplikować choremu bańki, kompresy; Laskawemu informatorowi aplikuje siarczysty policzek. (Dor)

2. (prawn.) eine juristische Praxis durchmachen: aplikować w sądzie

3. applizieren (eine Verzierung aus Stoff, Leder, dünnem Material auf ein Gewebe aufnähen) ( $\uparrow$ applizieren 3): wzór aplikowany na bluzke 
wäre es aufgrund des heutigen Gebrauchs als Inklusion einzustufen, bei der das deutsche Verb weniger Bedeutungsvarianten aufweist als das polnische, es liegt also ein Divergenzverhältnis vor. Das polnische Verb wird nämlich nicht nur in der ersten Bedeutungsvariante des deutschen Verbs verwendet:

Nie można wedlug mnie bez przerwy aplikować dyskursów i terminów wziętych z Zachodu, takich jak konceptualizm czy minimalizm, do opisania zjawisk dziejacych się w Polsce w tamtym czasie. ${ }^{1}$

Relacyjna koncepcja osobowości i środowiska życia, a także koncepcja rozwoju jako serii kryzysów, pozwalaja aplikować je jako podstawowe zalożenia teoretyczne stanowiące podstawę konceptualizacji diagnozy resocjalizacyjnej [...]. ${ }^{2}$

es erscheint zudem in zwei weiteren Bedeutungsvarianten:

1. jdm. etw. aufzwingen

Nie sądzę natomiast, aby rozwiazanie, które traktujemy jako epizodyczne i które budzi w petni uzasadnione kontrowersje, aplikować naszym partnerom z Unii Europejskiej. ${ }^{3}$

2. sich (um etw.) bewerben (womöglich in Anlehnung an das Englische):

Podczas posiedzenia $w$ dniu 5 czerwca zarząd województwa przyjąt uchwałe dotyczaca gmin, które będa mogly aplikować o środki do Funduszu Wsparcia. ${ }^{4}$

Ähnlich wären auch die Bedeutungsvarianten bei dem Wortpaar deklarieren vs. deklarować zu ergänzen. Im Gegensatz zu dem deutschen Verb wird das polnische Verb auch reflexiv gebraucht und es aktualisiert in dieser Verwendungsweise folgende zusätzliche Bedeutungsvarianten: ${ }^{5}$

1. sich für/gegen etw. aussprechen

Nie idzie o to, by deklarować się po stronie rynku lub ludu [...]. ${ }^{6}$

2. sich bereit erklären

To zależy od odpowiedzi, panie przewodniczacy, więc nie chciatbym z góry tutaj deklarować się, żeby później nie dotrzymać stowa. ${ }^{7}$

${ }^{1} \mathrm{http}: / /$ nkjp.uni.lodz.pl/ParagraphMetadata?pid=6d32fd77620f3819cdb217573c2af4a3\&match_start=262\&match_end=271\&wynik=26\#the_match; Stand vom 27.02.2017.

${ }^{2}$ http://nkjp.uni.lodz.pl/ParagraphMetadata?pid=5c409c7e4f0066e857615843c0ae77f8\&match_start $=108 \&$ match_end $=117 \&$ wynik=33\#the_match; Stand vom 27.02.2017.

${ }^{3}$ http://nkjp.uni.lodz.pl/ParagraphMetadata?pid=7729011d9f7319eff7f54286a1f97bb8\&match start=120\&match end=129\&wynik=80\#the match; Stand vom 27.02.2017.

${ }^{\overline{4}}$ http://nkjp.uni.lodz.pl/ParagraphMetadata?pid=7f4fbe0e384948c304aad6a1 cf67dd42\&match_start $=105 \&$ match_end $=114 \&$ wynik=42\#the_match; Stand vom 27.02.2017.

${ }_{5}^{5}$ Vgl. http://sjp.pwn.pl/szukaj/deklarowa\%C4\%87.html; Stand vom 28.02.2017.

${ }^{6} \mathrm{http}: / /$ nkjp.uni.lodz.pl/ParagraphMetadata?pid=9299ae0742c6eaef6bb810bc15f51976\&match start $=249 \&$ match end $=263 \&$ wynik $=3 \#$ the match; Stand vom 28.02.2017.

${ }^{7}$ http://nkjp.uni.lodz.pl/ParagraphMetadata?pid=c1f4c9819dd6632b5ccc2975b651a140\&match_start=79\&match_end=93\&wynik=10\#the_match; Stand vom 28.02.2017. 
3. veralt. offiziell um die Hand einer Frau anhalten

Auch bei dem Wortpaar delegieren - delegować scheint ebenfalls nicht mehr eine Inklusion vorzuliegen, bei der das deutsche Verb mehr Bedeutungsvarianten aufweist als das polnische, wie dem Wörterbucheintrag entnommen werden könnte:

10)

dt. delegieren (Lipczuk et al. 1995: 52)

1. delegować, oddelegować, wysyłać (kogoś jako swego przedstawiciela): $j d n$. zu einer Konferenz, zum Studium, in ein Komitee, in einen anderen Betrieb delegieren (nieco szersze znaczenie niż delegować)

2. przekazać (komuś swoje prawa, obowiązki itp.); zrzucać odpowiedzialność na innych: $E r$ versteht es, Arbeit zu delegieren. Diese Verantwortlichkeit kann ich nicht delegieren.

\section{pl. delegować}

delegieren (1), entsenden, schicken, abordnen, deputieren: Centrala Handlu Zagranicznego deleguje za granicę swych przedstawicieli.

Das polnische Verb wird im Gegenwartspolnischen ebenso in der zweiten Bedeutungsvariante des deutschen Verbs verwendet, im Sinne von 'übertragen', etwa: ${ }^{8}$

Musisz zaczać delegować więcej prac i oplacać pomocników [...]. ${ }^{9}$

Bei der Verifizierung des gegenwärtigen Bedeutungsinhalts der analysierten Verben fällt zudem auf, dass Bedeutungsvarianten, die beiden Sprachen gemeinsam sind, nicht immer für wahre Freunde zu erachten sind. Während etwa $a b$ dykować im Polnischen in der Bedeutung 'abdanken' auch gegenwärtig häufig verwendet wird, ist abdizieren im Gegenwartdeutschen heutzutage nicht mehr anzutreffen. Es wäre somit als ein Verstoß gegen die sprachliche Norm zu werten, würde man dieses Verb als Pendant des polnischen verwenden. Das Gleiche betrifft das Verb assekurieren, wie der Verwendungshäufigkeit im Digitalen Wörterbuch der Deutschen Sprache entnommen werden kann.

Aufgrund der Tatsache, dass das Verb im Gegenwartsdeutschen nicht mehr usuell ist, könnte hier schwerlich von einem wahren Freund die Rede sein, sondern vielmehr von einem formalen falschen Freund.

${ }^{8}$ Vgl. http://sjp.pwn.pl/szukaj/delegowa\%C4\%87.html; Stand vom 28.02.2017.

${ }^{9}$ http://nkjp.uni.lodz.pl/ParagraphMetadata?pid=00c6501344bebd408d3ec66e67a7ad42\&match_start=23\&match_end=32\&wynik=12\#the_match; Stand vom 28.02.2017. 
assekurieren - Verlaufskurve

Basis: DWDS-Zeitungskorpus (ab 1945)

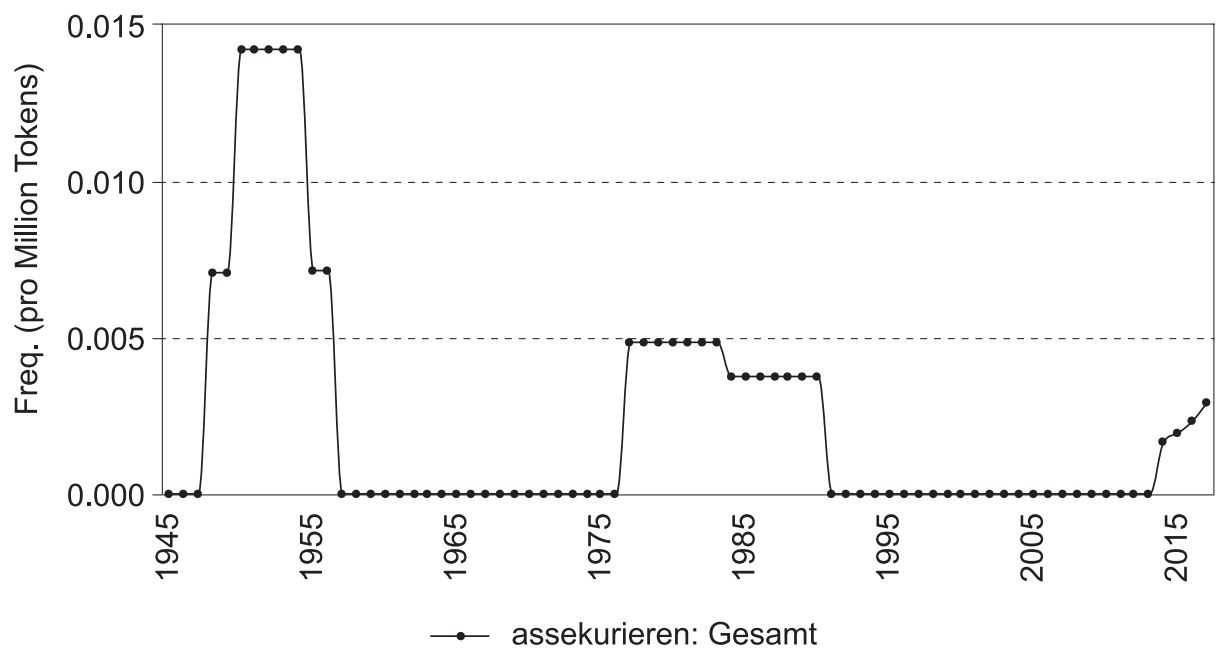

Abb. 1. Worthäufigkeit von assekurieren

\section{Fazit ${ }^{10}$}

Unterschiedliche soziale, politische, wirtschaftliche, kulturelle oder historische Hintergründe können dazu führen, dass formal ähnliche Wörter, die in zwei Sprachen auch die gleiche Herkunft haben, in ihren Wortinhalten nicht übereinstimmen. Diese Nichtübereinstimmung muss jedoch nicht nur auf unterschiedliche Sprachen, sondern auch auf unterschiedliche Sprachstufen bezogen sein. Bei falschen Freunden können somit nicht lediglich Fälle von synchronisch-interlingualer, sondern auch von diachronisch-intralingualer Nichtübereinstimmung vorliegen (vgl. Grosse 1998: 361).

Vor diesem Hintergrund gilt es die einschlägigen Tautonymenwörterbücher zu aktualisieren, bei didaktischer Aufbereitung von Lehr- und Lernmaterialien sollten wiederum in erster Linie Wortpaare berücksichtigt werden, die häufig vorkommen, damit Formulierungen wie der aus einer in Zweitsprache Deutsch verfasster Diplomarbeit vorgebeugt wird:

In Verbindung mit der Geschichte spielen die Stereotypen eine doppelte Rolle. Einerseits intrigieren sie, andererseits schließen sie sich gegenseitig aus.

Darin erfuhr das im Polnischen häufig gebrauchte Verb intrygować aufgrund der Formähnlichkeit mit dem deutschen intrigieren eine zum kommunikativen Fehler führende Bedeutungserweiterung.

${ }^{10} \mathrm{https} / / /$ www.dwds.de/wb/assekurieren; Stand vom 28.02.2017. 
Angesichts des Bedeutungswandels, wie an einigen der analysierten Wortbeispiele veranschaulicht, bestätigt sich die Beobachtung Hills, wonach Wörter und Menschen eine Wesensverwandtschaft verbinde (vgl. Hill et al. 2000: 88; zit. nach Ludewig 2005: 67). Im Zuge des Bedeutungswandels, aber auch der Veränderungen im Sprachgebrauch können nämlich aus wahren Freunden falsche werden und umgekehrt.

\section{Literatur}

Bawej, Izabela (2014): Muttersprachliche Interferenz im Lichte der kontrastiven Fehleranalyse am Beispiel Polnisch-Deutsch. Bydgoszcz.

Chodacka, Mariola / Schöffel, Heinz-Uwe (2000): Es gibt auch ,echte Freunde “ in der Fremdsprache. In: Studia Germanica Posnaniensia XXVI. S. 169-182.

Grosse, Ernst Ulrich (1998): Zwischen Linguistik und Landeskunde: die "faux amis culturels “. In: Französich heute 29. S. 359-378.

Janikowski, Przemysław / Krzywda, Joanna (2011): Fatszywi przyjaciele od lat (Próba systematyzacji badań). In: Fast, Piotr / Car, Anna / Osadnik, Wacław M. (Hrsg.): Historyczne oblicza przekładu. Katowice. S. 115-135. [Online unter: http://www.academia.edu/6725951/ Fa\%C5\%82szywi_przyjaciele_od_lat_pr\%C3\%B3ba_systematyzacji_bada\%C5\%84_; Stand vom 13.02.2017].

Kosturek, Kamila (2013): Falsche Freunde - ein Hindernis im Fremdsprachenlernprozess nur in der Theorie oder auch in der Praxis? In: Glottodidactica XL/1. S. 91-103.

Księżyk, Felicja (2015): Kollokationen im Zivilrecht Polens in den Jahren 1918-1945 mit besonderer Berücksichtigung der deutschsprachigen Zivilgesetzbücher. Frankfurt am Main.

Kuczyński, Ernest (2003): Ein Diskurs zu geschichtlichen, terminologischen und definitorischen Fragen der ,,falschen Freunde des Übersetzers “. In: Orbis Linguarum, Vol. 24. S. 255-280.

Kuczyński, Ernest (2005): Interferenzträchtige Stolpersteine bei der Übersetzung. Das Wesen und die Problematik der ,falschen Freunde des Übersetzers". Włocławek.

Lietz, Gero (2006): , Deutsch-polnisches Kriminalistenseminar “. ,Falsche Freunde' im Fremdsprachenunterricht. In: Convivium. Germanistisches Jahrbuch Polen. S. 321-345.

Lipczuk, Ryszard (1990): Mały słownik tautonimów niemiecko-polskich. Warszawa.

Lipczuk, Ryszard (1992): Internacjonalizmy a „fatszywi przyjaciele tlumacza”. In: Maćkiewicz, Jolanta / Siatkowski, Janusz (Hrsg.): Język a kultura 7. Wrocław. S. 135-143.

Lipczuk, Ryszard; Bilut-Homplewicz, Zofia / Kątny, Andrzej / Schatte, Christoph (1995): Niemiecko-polski stownik tautonimów. Warszawa.

Lisiecka-Czop, Magdalena (2007/2008): „Prawdziwi przyjaciele tlumacza“ a dydaktyka i praktyka przekładu. In: Rocznik Przekładoznawczy. Studia nad teorią, praktyką i dydaktyką przekładu. S. 271-282.

Ludewig, Petra (2005): Korpusbasiertes Kollokationslernen. Computer-Assisted Language Learning als prototypisches Anwendungsszenario der Computerlinguistik. Frankfurt am Main.

URL 1: http://lipczuk.univ.szczecin.pl/ArtFA.htm; Stand vom 13.02.2017.

Germanica Wratislaviensia 143, 2018

(C) for this edition by CNS 